\title{
The effects of expansion line opacity in accretion disks
}

\author{
G. Shaviv ${ }^{1,2}$ and R. Wehrse ${ }^{1}$ \\ 1 Institut für Theoretische Astrophysik Tiergartenstrasse 15, 69121 Heidelberg, Germany \\ 2 On leave from Department of Physics and Asher Space Research Institute, Technion, 32000 Haifa, Israel \\ e-mail: gioras@physics.technion.ac.il
}

Recieved 10 June 2005 / Accepted 19 July 2005

\begin{abstract}
We estimate the effect of the shear flow in accretion disks on the radiation field. We apply the recent results of Wehrse et al. (2003, A\&A, 401, 43) for the expansion line opacity in shear flows. We use an approximation and derive an approximate equation for the radiation field in an accretion disk in the diffusion approximation. We find that in the appropriate limit for the flow in disks:

- The effect depends on a product of three factors: the first depends solely atomic physics and hence on the composition of the accreted matter, the second depends on the density while the third depends solely on the shear flow.

- The radiation field in accretion disks around neutron stars, black holes and white dwarfs is strongly affected by the expansion line opacity.

- The radiation field is very non isotropic leading to significantly higher specific intensities in the vertical direction, a higher temperature gradient and increased susceptibility to convection and other instabilities.
\end{abstract}

Key words. disks, shear flow, expansion opacity

\section{Introduction}

Radiative transfer in moving media is affected by velocity gradients. The effect, dubbed "expansion opacity", was first evaluated by Karp et al. (1977). In the simplest model of an accretion disks the flow is Keplerian and hence possess a shear in the radial direction. Hence, a photon propagating inside the disk sees an anisotrpic shear flow. The enhanced effective line opacity, called expansion opacity, is very non isotropic. The main purpose of this letter is to expose the importance of this effect to the structure of accretion disks (here after AD).

\section{The expansion opacity}

\subsection{Effective extinction in a given direction}

Let $\boldsymbol{n}$ be a unit vector in the direction of the ray and $\boldsymbol{v}(\boldsymbol{x})$ a velocity field. Define the scalar "velocity gradient" as:

$w(\boldsymbol{n})=\boldsymbol{n} \cdot \nabla(\boldsymbol{n} \cdot \boldsymbol{\beta})=\boldsymbol{n} \frac{\partial \boldsymbol{\beta}}{\partial \boldsymbol{x}} \boldsymbol{n}=\sum_{i, j} n_{i} \frac{\partial \boldsymbol{\beta}}{\partial x_{j}} n_{j} ; \boldsymbol{\beta}=\frac{\boldsymbol{v}}{c}$.

In the general case of a moving media the radiative flux vector (to distinguish from the flux in a given direction $\boldsymbol{n}$ ) is given by (Wehrse et al., Paper II):

$\boldsymbol{F}_{w}\left(s_{0}, \xi\right)=\frac{4 \pi}{3} \frac{\partial B(T, \xi)}{\partial T}\left[\boldsymbol{X}_{\beta}(\xi)\right]^{-1} \nabla T$ where $\boldsymbol{X}_{\beta}(\xi)$ is a generalized extinction tensor namely:

$$
\left[\boldsymbol{X}_{\beta}(\xi)\right]^{-1}=\frac{3}{2 \pi} \int_{2 \pi}\left[\chi_{n}(\xi, w(\boldsymbol{n}))\right]^{-1} n_{i} n_{j} \mathrm{~d} \omega
$$

$\xi$ is the wavelength and $s_{0}$ the location. The static extinction coefficient $\chi_{0} \equiv \chi_{n}(\xi, w(\boldsymbol{n}) \rightarrow 0)$ is independent of direction and hence the static flux vector is:

$F\left(s_{0}, \xi\right)=\frac{4 \pi}{3} \frac{\partial B(T, \xi)}{\partial T}\left[\chi_{0}(\xi)\right]^{-1} \nabla T$

\subsection{Total effective opacity}

Clearly, $w=w(\boldsymbol{n})$ is a scalar function, the value of which depends on direction. Consequently, the effective opacity is a function of direction. On the other hand, the extinction $\boldsymbol{X}_{\beta}$ becomes a tensor. The deviation of the radiation vector, which is proportional to $\left[\boldsymbol{X}_{\beta}\right]^{-1} \nabla T$, from $\nabla T$ is important for induced circulation in the ADs and will be treated elsewhere. The total flux in direction $\boldsymbol{n}$ is obtained by integration over the solid angle and is:

$F_{\text {tot }}\left(s_{0}, w\right)=\frac{4 \pi}{3} \frac{\partial B(T)}{\partial T} \overline{\left[\chi_{n}(w)\right]^{-1}} \boldsymbol{n} \cdot \nabla T$

where

$\overline{\left[\chi_{n}(w)\right]^{-1}}=\int_{-\infty}^{\infty} G\left(s_{0}, \xi\right) \exp (\xi)\left[\chi_{n}(\xi, w)\right]^{-1} \mathrm{~d} \xi$ 
and

$G\left(s_{0}, \xi\right)=\left(\frac{\partial B\left(s_{0}, \xi\right)}{\partial T} / \frac{\mathrm{d} B(T)}{\mathrm{d} T}\right) \exp (\xi)$

\section{The effective opacity}

We consider here a general case of a series of infinitely sharp lines with a distribution in amplitude. Let the line density be:

$\rho(\xi, A)=\rho(\xi) f(A)$,

where $\rho(\xi)$ is the number density per unit logarithmic wavelength interval $\Delta \log \xi=1$ and $f(A)$ is the normalized line strength distribution. In this simplified picture we assume that the line strengths and line density are independent. The mean line strength is given by:

$\bar{A}=\int_{A} A f(A) \mathrm{d} A$.

The total line extinction is then written as: $\chi_{1}=\rho(\xi) \bar{A}$ and a new parameter $\Lambda$ is defined as

$\Lambda(\xi)=\frac{\chi_{1}(\xi)}{\chi_{\mathrm{c}}(\xi)}=\rho(\xi)\left(\frac{\bar{A}}{w}\right)\left(\frac{w}{\chi_{\mathrm{c}}(\xi)}\right)$

with

$\frac{\mathrm{d} \beta}{\mathrm{d} \tau_{\mathrm{c}}}=\frac{w}{\chi_{\mathrm{c}}(\xi)}, \quad \frac{\mathrm{d} \beta}{\mathrm{d} \tau_{1}}=\frac{w}{\rho(\xi) \bar{A}}$,

where $\mathrm{d} \tau_{\mathrm{c}}=\chi_{\mathrm{c}} \mathrm{d} s$ and $\mathrm{d} \tau_{1}=\chi_{1} \mathrm{~d} s$. We note that the $w(\boldsymbol{n})$ dependence in $\Lambda$ cancels out. $w / \chi_{\mathrm{c}}$ is the Doppler spread $\Delta \xi_{\mathrm{D}, \mathrm{c}}$ over a mean free path $1 / \chi_{c}$ of a photon in the continuum and $w / \bar{A}$ is the line density $\rho(\xi)$ times the velocity change $\Delta \xi_{\mathrm{D}, 1}$ over one mean free path of a photon in an average spectral line. Once $f(A)$ is given, the effective opacity can be calculated. The result under the present assumptions can be cast into the following general form:

$\frac{\chi_{\mathrm{eff}, n}(\xi, w)}{\chi_{\mathrm{eff}, 0}(\xi)}=1+\Lambda(\xi) S\left(\frac{w}{\bar{A}}\right)$

where the function $S(w / \bar{A})$ depends on the assumption about the line distribution.

The function $S(w / \bar{A})$ was calculated by Wehrse et al. (2003, Paper III) for several assumptions about the distribution of lines. For example, in the case of Poisson distributed infinitely sharp lines it has the form:

$S(x)=x\left(1-\exp \left(-\frac{1}{x}\right)\right) ; \quad x=w / \bar{A}$.

Under these conditions, the effect is negligible for $w / \bar{A} \leq 0.1$ and reaches saturation for $w / \bar{A} \geq 10$. The second parameter $\Lambda$ may be as high as few $\times 10^{5}$ which means that the saturation level of the effect due to velocity gradient can be as high as $10^{5}$ times the continuum.

For $w \ll 1$ we find (Wehrse et al., Paper III) that

$\chi_{\mathrm{eff}}^{-1} \approx \frac{1}{\chi_{\mathrm{R}}}\left(1-\left|\eta_{2}\right| w^{2}\right)=\frac{1}{\chi_{\mathrm{R}}}\left(1-\frac{1}{\rho^{2}} \eta_{\mathrm{norm}} w^{2}\right)$ where $\eta_{2}$ is given by (Wehrse et al., Paper II, Eq. (21)) by:

$\eta_{2}\left(s_{0}\right)=\frac{1}{2} \bar{\chi}_{\mathrm{R}}\left(s_{0}\right) \int_{-\infty}^{\infty}\left(\frac{G\left(s_{0}, \xi\right)}{\chi(\xi)}\right) \frac{\partial^{2}}{\partial \xi^{2}}\left[\chi(\xi)^{-2}\right] \mathrm{d} \xi$.

We find it is advantageous to remove the $\rho$ dependence and define $\eta_{2}=\rho^{-2} \eta_{\text {norm }}$. We write now

$F\left(s_{0}, \xi, w\right)=\left[\frac{\chi_{n}(\xi, w)}{\chi_{0}(\xi)}\right]^{-1} F\left(s_{0}, \xi\right)$

and we write similarly for the diffusion in the moving media:

$F_{w}\left(s_{0}, \xi\right)=\frac{4 \pi}{3} \frac{\partial B(T, \xi)}{\partial T}\left[\chi_{\beta}(\xi)\right]^{-1} \nabla T$

where:

$F\left(s_{0}, \xi, w\right)=2 q\left(s_{0}, \xi, \boldsymbol{n}\right) \int \mathrm{d} s \exp \left(-\frac{1}{w} \int_{\xi-w_{s_{0}}}^{s} \chi(\xi) \mathrm{d} \xi\right)$

and $2 q=(\partial B / \partial T) \boldsymbol{u} \cdot \nabla T$.

Define now an effective opacity as:

$\chi_{\mathrm{eff}}^{-1}=\int_{0}^{\infty} \exp \left(-\frac{1}{w} \int_{\xi-w_{s_{0}}}^{\xi} \chi(\xi) \mathrm{d} \xi\right) \mathrm{d} s=\frac{U\left(\eta_{2}, w^{2}\right)}{\chi_{\mathrm{R}}}$,

where $U\left(\eta_{2}, w^{2}\right)$ is the correction function. $\eta_{2}$ should be calculated from a given list of lines, if this is given, or else under the assumption of a Poisson Point Process for example. Note that $\eta_{2} \leq 0$ for spectral lines.

The function $U\left(\eta_{2}, w^{2}\right)$ is given in Fig. 6 of Wehrse et al. (2003) in the case of Poisson distributed spectral lines and for a range of parameters for the spectral lines. The sensitivity to the parameters of the spectral lines (as is clear from the figure) is small and will be neglected here. The general behavior of $U\left(\eta_{2}, w^{2}\right)$ is as follows: for $\log w \leq-11$ there is no significant effect, while for $\log w \approx 9$ the function reaches saturation at about $10^{2}-10^{4}$ times the value at $\log w \rightarrow-\infty$ (cf. Table 2 ibid.). In the limit of very large velocity gradients we get:

$\chi_{\mathrm{eff}}(\xi, w \rightarrow \infty)=\chi_{\mathrm{c}}+\rho_{0} A_{0}$

for Poisson distributed lines of equal strength $A_{0}=\bar{A}$ with Lorentz profile and Dirac $\delta$ line profile.

In the limit of small velocity gradients we can write $U\left(\eta_{2}, w^{2}\right)=1-\rho^{-2}\left|\eta_{\text {norm }}\right| w^{2}$.

\section{The diffusion flux}

Clearly, the diffusion flux is very non-isotropic. Expanding the flux in the direction $\boldsymbol{n}$ in terms of $w$ yields:

$$
\begin{aligned}
& F_{n}\left(s_{0}, \xi, w(\boldsymbol{n})\right)=\left[\frac{\chi_{\boldsymbol{n}}(\xi, w(\boldsymbol{n}))}{\chi_{0}(\xi)}\right]^{-1} F_{n}\left(s_{0}, w(\boldsymbol{n})\right) \\
& =\left[1+f_{1}(\xi) \boldsymbol{\Psi}_{1}(w(\boldsymbol{n}))+f_{2}(\xi) \boldsymbol{\Psi}_{2}(w(\boldsymbol{n}))\right] F_{n}\left(s_{0}, w(\boldsymbol{n})\right)
\end{aligned}
$$

where index $\mathrm{n}$ implies flux in the $\boldsymbol{n}$ direction and

$f_{1}(\xi)=-\frac{\partial}{\partial \xi}\left(\frac{1}{\chi_{0}(\xi)}\right), \quad f_{2}(\xi)=\frac{\partial^{2}}{\partial \xi^{2}}\left(\frac{1}{\chi_{0}(\xi)}\right)^{2}$. 
Note the particular form of the expansion, namely functions of $\xi$ times functions of $w$. To obtain the flux vector one has to integrate Eq. (21) over the solid angle to get (cf. Wehrse et al., Paper IV):

$\boldsymbol{F}_{w}\left(s_{0}, \xi\right)=\left[\boldsymbol{E}+\frac{1}{5} f_{1}(\xi) \boldsymbol{\Psi}_{1}+\frac{1}{70} f_{2} \boldsymbol{\Psi}_{2}\right] \boldsymbol{F}\left(s_{0}, \xi\right)$

with the matrices:

$$
\begin{aligned}
\boldsymbol{\Psi}_{1}= & \operatorname{Tr}\left(\frac{\partial \boldsymbol{\beta}}{\partial \boldsymbol{x}}\right)_{s} \boldsymbol{E}+\left(\frac{\partial \boldsymbol{\beta}}{\partial \boldsymbol{x}}\right)_{s} \\
\boldsymbol{\Psi}_{2}= & \left(\operatorname{Tr}\left(\frac{\partial \boldsymbol{\beta}}{\partial \boldsymbol{x}}\right)_{s}\right)^{2} \boldsymbol{E}+2 \operatorname{Tr}\left(\frac{\partial \boldsymbol{\beta}}{\partial \boldsymbol{x}}\right)_{s}^{2} \boldsymbol{E} \\
& +4 \operatorname{Tr}\left(\frac{\partial \boldsymbol{\beta}}{\partial \boldsymbol{x}}\right)_{s}\left(\frac{\partial \boldsymbol{\beta}}{\partial \boldsymbol{x}}\right)_{s}+8\left(\frac{\partial \boldsymbol{\beta}}{\partial \boldsymbol{x}}\right)_{s}^{2}
\end{aligned}
$$

and

$\left(\frac{\partial \boldsymbol{\beta}}{\partial \boldsymbol{x}}\right)_{s}=\frac{1}{2}\left[\left(\frac{\partial \boldsymbol{\beta}}{\partial \boldsymbol{x}}\right)+\left(\frac{\partial \boldsymbol{\beta}}{\partial \boldsymbol{x}}\right)^{T}\right]$

namely, only the symmetric part in the Jacobian $\partial \beta / \partial \boldsymbol{x}$ contributes to the effect.

\section{Application to disk shear flow}

The zeroth order disk structure is a Keplerian shear flow, namely

$\beta=\frac{\boldsymbol{v}}{c}=\sqrt{\frac{2 G M}{c^{2} r}}\left(-\frac{y}{r}, \frac{x}{r}, 0\right)=\sqrt{\frac{R_{\mathrm{sh}}}{r}}\left(-\frac{y}{r}, \frac{x}{r}, 0\right)$,

so that $\boldsymbol{\Psi}_{k}$ for $k=1,2$ can be calculated for the AD. $R_{\mathrm{sh}}$ is the Schwarzchid radius. First we note that there are 4 main directions in which a moving photon does not experience any effect: up and down, radially outward and inward. In all other directions the photon sees a velocity shear. Since the effect depends on $w^{2}$ there is no difference between the outgoing or ingoing photon. Once the velocity field is given, we can determine whether $w$ is small or large, namely what order of effect we expect. Next, for Keplerian shear flow $\operatorname{Tr}(\partial \boldsymbol{\beta} / \partial \boldsymbol{x})=0$ and the term with $f_{1}$ vanishes upon integration over wavelength (it exists only in the monochromatic case). For ADs,

$w \sim 1 /\left(2 R_{\mathrm{sh}}\right)\left(R_{\mathrm{sh}} / r\right)^{3 / 2}$.

Returning to Eq. (14) we have approximately

$\chi_{\mathrm{eff}}^{-1} \approx \frac{1}{\chi_{\mathrm{R}}}\left(1-\rho^{-2} \frac{\left|\eta_{\mathrm{norm}}\right|}{280 R_{\mathrm{sh}}^{2}}\left(\frac{R_{\mathrm{sh}}}{r}\right)^{3}\right)$.

The estimate for $\eta_{\text {norm }}$ depends on the assumption on the shape of the lines. Typical values of $\eta_{\text {norm }}$ are shown in Fig. 1 . The $\eta_{\text {norm }}$ was calculated for solar composition material and various number of lines per $\Delta \log \xi=1$ and Doppler widths (which is equivalent to the temperature). We find extremely large values of $\eta_{\text {norm }}$ over a large range of parameters, namely it is very frequent that $\eta_{\text {norm }}$ is extremely large. As the temperature decreases and with it the Doppler width the effect increases. Clearly, the effect increases with the number of lines

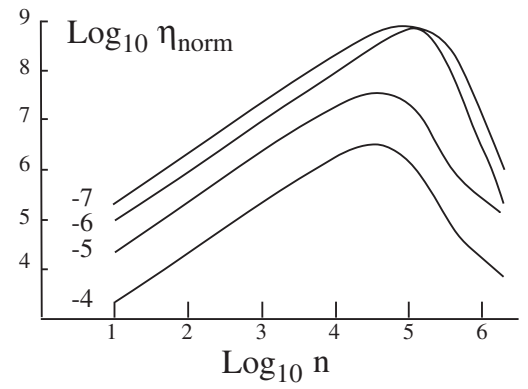

Fig. 1. The dependence of $\left|\eta_{\text {norm }}\right|$ on the number of lines per $\Delta \log \xi=1$ and on the Doppler width. The logarithm of the Doppler width is shown on the left side of the corresponding curve.

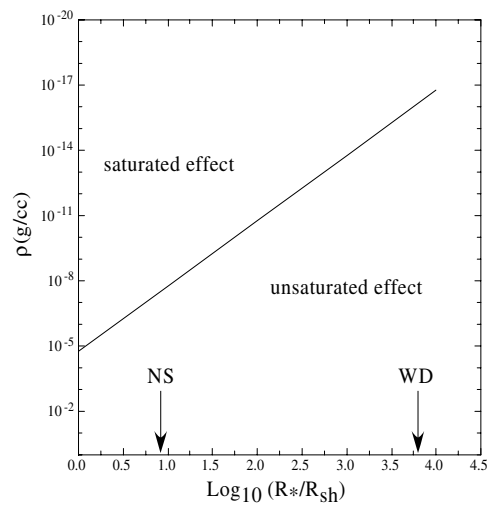

Fig. 2. The critical density for saturation of the expansion opacity as a function of the location in the disk for an accreting object of $1 M_{\odot}$.

but for any given Doppler width, with sufficiently large number of lines, the lines start to overlap each other and decreases the effect.

The effect contains three factors, the density, the composition and the geometry. For given density and composition the effect is largest near the surface of the accreting star or the Schwarzchild radius in the case of a black hole. The effect is important for

$\left|\eta_{\text {norm }}\right| \approx 6.08 \times 10^{12} x^{3} \rho^{2}\left(M / M_{\odot}\right)^{-2}$,

where $x=r_{*}$ is the radius of the object in units of the Schwarzschild radius for a $1 M_{\odot}$ object. For a $1 M_{\odot}$ compact Neutron star $x \approx 6.8$. Hence the condition becomes:

$\left|\eta_{\text {norm }}\right|_{\text {bh,ns,wd }} \geq\left(6.08 \times 10^{12}, 1.9 \times 10^{15}, 5.5 \times 10^{23}\right) \rho^{-2}$.

We find that for most parameter combinations the effect of the expansion opacity reaches saturation, namely it reaches the maximum (cf. Eq. (20) is the total line absorption $\rho_{0} A_{0}$ which can be as high as $10^{3}$ times the Thomson continuum.

\section{The effect on the structure of the disk}

The enhancement of the opacity is very anisotropic. In Fig. 3 we show the geometrical factor $\boldsymbol{n}(\partial \boldsymbol{\beta} / \partial \boldsymbol{x}) \boldsymbol{n}$ as a function of $\theta, \varphi$ at the location $(1,0,0)$. In reality, this term is multiplied by $\eta_{2} \gg 1$ which accentuates the unisotropy.

Because of the extremely large $\eta_{\text {norm }}$, saturation is reached very quickly and radiation escapes in the directions of smallest 


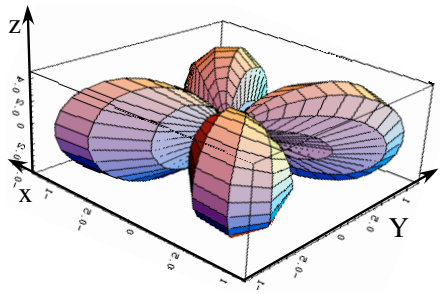

Fig. 3. The dependence of the shear as a function of direction.

opacity. Namely, $I_{\text {eff }}=F_{0} / \Delta \Omega$ where $\Delta \Omega \ll 4 \pi$ is the solid angle in the direction of unchanged opacity, $F_{0}$ is the would be radiative flux if no expansion opacity effects existed and $I_{\text {eff }}$ is the specific intensity.

The extremely large $\eta_{\text {norm }}$ plus the very anisotropic $\boldsymbol{n}(\partial \boldsymbol{\beta} / \partial \boldsymbol{x}) \boldsymbol{n}$ leads to a situation where the effect enters already at very small shear. In other words, already in a very small deviation from the primary directions where the shear vanishes, the effect is at its maximum value so that $\Delta \Omega$ is extremely small.

As a consequence, the temperature gradient increases and leads right away to convective instability. This instability can be the source of macroscopic viscosity in the above ADs. The temperature gradient is given by

$\frac{\mathrm{d} B_{w}(T)}{\mathrm{d} z}=-\frac{3}{4} F \chi_{\mathrm{eff}} \rho=\frac{\mathrm{d} B_{0}(T)}{\mathrm{d} z}\left(\frac{\chi_{\mathrm{eff}}}{\chi_{0}}\right)$

where the indices $w, 0$ refer to the case with velocity shear and the case without velocity shear respectively.

Clearly, the problem of an ideal ADs with only continuum opacity remains. The shear in the velocity has no effect when spectral lines are absent.

In the particular case of ADs we have to substitute the divergence of the total flux vector into the energy equation. Under the present approximation we return to Eq. (23) which transforms to

$\nabla^{2} B_{w}=\frac{Q(r, z)-\nabla B_{w} \cdot \nabla f(r, z)}{1-\left(\left|\eta_{2}\right| / R_{\mathrm{sh}}\right)\left(R_{\mathrm{sh}} / r\right)^{3}}$ where $Q(r, z)$ is the energy source and $f(r, z)$ a known function of position. Since the total energy radiated away is essentially fixed (for fixed $\dot{m}$ ) the increase in $\chi_{\text {eff }}$ increases $\mathrm{d} T / \mathrm{d} z$ but does not increase the location of the surface and the overall averaged $T_{\text {eff }}$. (Saturation prevents the singularity.)

\section{Conclusions}

While the velocity gradients keep the effect in the limit of low $w$, the large $\eta_{2}$ drives the effect into saturation.

We assumed that averaging over angle is plausible (as first order). The strong anisotropy calls for an improved treatment of the radiation field. This extension is underway and will be reported in due course along with the stability analysis.

The limb darkening effect of the disk must be very peculiar with significant consequences to the estimated total luminosity of disks observed at various angles. The expected structure of the atmosphere with non isotropic effective opacity differs significantly from hitherto assumed structure (work in progress).

Magnetic fields, which were ignored here, add to the velocity fields and hence broaden the lines sooner without affecting the global Keplerian flow and general effect.

Acknowledgements. G.S. acknowledges partial support by the Israel Science Foundation and by the DFG (Sonderforschungsberiech $359 / \mathrm{C} 2)$

\section{References}

Karp, A. H., Lasher, G., Chan, K. L., \& Salpeter, E. E. 1977, ApJ, 161, 214

Wehrse, R., Bascheck, B., \& von Waldenfels, W. 2000a, A\&A, 359, 780 (Paper I)

Wehrse, R., Bascheck, B., \& von Waldenfels, W. 2000b, A\&A, 359, 788 (Paper II)

Wehrse, R., Bascheck, B., \& von Waldenfels, W. 2002, A\&A, 390, 1141 (Paper III)

Wehrse, R., Bascheck, B., \& von Waldenfels, W. 2003, A\&A, 401, 43 (Paper IV) 